\title{
Constant-Voltage Steps in Josephson Tunneling
}

\author{
Chong Der Hu \\ Department of Physics, National Taiwan University, Taipei, Taiwan R.O.C.
}

(Received June 25, 1997)

\begin{abstract}
We studied the ac Josephson effect. In the resistively shunted junction model (RSJ) the phase difference is expanded in Fourier series. The Fourier components can be evaluated by solving the equations of motion. Truncation is applied to deal with the infinite products and series of Bessel functions. We calculate the constant-voltage steps and find that they are complicate functions of the $a c$ power input.
\end{abstract}

KEYWORDS: Josephson tunneling, nonlinear phenomena

\section{§1. Introduction}

The ac Josephson effect has been an active field of study $^{1)}$ since it was discovered by Shapiro. ${ }^{2)}$ The most prominent feature in the $I-V$ characteristic curve is the constant-voltage steps. They appear at $2 e V_{d c}=n \hbar \omega$ where $\omega$ is the frequency of the $a c$ bias power. The step size varies with the square root of the $a c$ bias power in an oscillatory manner. In some cases it has a Bessel function behavior. Subharmonic steps had also been found. Usually, one can compute the step sizes with the voltagesource model ${ }^{3)}$ and indeed, find that they vary with the $a c$ bias voltage as Bessel functions. However, many experimental data do not show this kind of behavior. ${ }^{3)}$ This discrepancy is attributed to the fact that the voltage across the junction is not easily maintained. Alternatively, the resistively shunted junction model (RSJ) $\left.{ }^{4}\right)$ can be used to analyze this phenomenon. Since it involves a nonlinear differential equation, simulation was often used ${ }^{5)}$ and obtained results consistent with experiments. Here, we attempt to provide a different point of view. We proposed an analytical solution and from which the step sizes are computed. It can be shown clearly how the step sizes are related to the $a c$ power input, as well as other parameters such as the resistance, capacitance, frequency and supercurrent amplitude. We believe this method is able to provide additional insight to Josephson tunneling. Furthermore, since the equation of motion of the RSJ model is the same as that of a dissipative pendulum under external forces, our method is useful for studying nonlinear dynamics. In $\S 2$ we derive the formalism. In $\S 3$ the step sizes are computed. The conclusion is given in $\S 4$.

\section{§2. Derivation of Phase}

The RSJ model gives the following equations of motion for the Josephson junction with a current source:

$$
\dot{\phi}=2 e V(t)
$$

and

$$
\frac{C}{2 e} \ddot{\phi}+\frac{1}{2 e R} \dot{\phi}+I_{J} \sin \phi=I_{d c}+I_{a c} \sin \omega t
$$

where $I_{J}$ is the supercurrent amplitude, $C$ and $R$ are the capacitance and resistance of the junction, $I_{d c(a c)}$ is the $d c(a c)$ bias current, and $\hbar$ was set to 1 . Equation (2) is a nonlinear equation. Up to now, most efforts of solving this equation use numerical simulation. ${ }^{6)}$ Here, we propose an analytical solution. Before doing so, we would like to outline some well-known mathematical properties of the solution ${ }^{7)}$ and thus, illustrate the motivation of formulating the solution below. The most obvious question to ask is whether there exists a periodic solution. If there is, then its frequency should be commensurable to $\omega$ and hence, can be expanded in a Fourier series. If such series is substituted into eq. (2), then, in the presence of $I_{d c}$, a term linear in $t$ is required. Its coefficient gives a new frequency due to the nonlinearity. Thus, the following expression is proposed:

$$
\begin{aligned}
\phi= & \phi_{0}+\omega_{0} t+\sum_{k, l} a_{k l} \sin \left(k \omega+l \omega_{0}\right) t \\
& +\sum_{m, n} b_{m n} \cos \left(m \omega+n \omega_{0}\right) t
\end{aligned}
$$

where $\phi_{0}$ is related to initial conditions and $\omega_{0}$ is to be determined. In general, the solution in eq. (3) is not periodic. It has some similarity with the one proposed by Hasselberg et $a l^{8)}$ and also ref. 9 for the voltage-biased case. However, the difference is profound. In the voltagebiased circuit, $\omega_{0}$ is equal to $2 e V_{d c}$ where $V_{d c}$ is the applied $d c$ voltage. In the present case, $\omega_{0}$, though related to $I_{d c}$, has to be computed. If $\omega_{0}$ is commensurable to $\omega$, then the solution is periodic. Higher harmonics and subharmonic components appear. As we shall see this is when the constant-voltage steps appear.

Substituting eq. (3) into eq. (2) and using the relations ${ }^{10)}$

$$
\exp (i z \sin \theta)=\sum_{m} J_{m}(z) \mathrm{e}^{i m \theta}
$$

and

$$
\exp (i z \cos \theta)=\sum_{n} i^{n} J_{n}(z) \mathrm{e}^{i n \theta}
$$

we get 


$$
\begin{aligned}
& \left(r \omega+s \omega_{0}\right)^{2} a_{r s}+\frac{\left(r \omega+s \omega_{0}\right)}{R C} b_{r s}+\frac{2 e I_{a c}}{C} \delta_{r 1} \delta_{s 0} \\
& =\frac{2 e I_{J}}{C} \sum_{\alpha, \beta} \cos \left(\sum \beta_{m n} \pi / 2+\phi_{0}\right)\left[\prod_{k, l} J_{\alpha_{k l}}\left(a_{k l}\right)\right]\left[\prod_{m, n} J_{\beta_{m n}}\left(b_{m n}\right)\right], \\
& \left(r \omega+s \omega_{0}\right)^{2} b_{r s}-\frac{\left(r \omega+s \omega_{0}\right)}{R C} a_{r s} \\
& =\frac{2 e I_{J}}{C} \sum_{\alpha, \beta} \sin \left(\sum \beta_{m n} \pi / 2+\phi_{0}\right)\left[\prod_{k, l} J_{\alpha_{k l}}\left(a_{k l}\right)\right]\left[\prod_{m, n} J_{\beta_{m n}}\left(b_{m n}\right)\right]
\end{aligned}
$$

and

$$
\frac{\omega_{0}}{2 e R}=I_{d c}-I_{J} \sum_{\alpha, \beta} \sin \left(\sum \beta_{m n} \pi / 2+\phi_{0}\right)\left[\prod_{k, l} J_{\alpha_{k l}}\left(a_{k l}\right)\right]\left[\prod_{m, n} J_{\beta_{m n}}\left(b_{m n}\right)\right] .
$$

The summations of $\alpha$ and $\beta$ mean to sum over all possible integer values. In eqs. (6)-(8) $\alpha$ and $\beta$ are under following constraints:

$$
\sum_{k, l, m, n}\left(\alpha_{k l} k+\beta_{m n} m\right)=r
$$

and

$$
\sum_{k, l, m, n}\left(\alpha_{k l} l+\beta_{m n} n\right)=s
$$

There are infinite series and products of Bessel functions in eqs. (6)-(8). We can truncate them by neglecting Bessel functions with large indicies and arguements with subscripts large in magnitude. This approximation is based on the fact that $a_{k l}$ and $b_{m n}$ are small in magnitudes if any one of the subscripts is large in magnitude. More details were given in ref. 9. If only the first few terms were kept, we have

$$
\begin{aligned}
a_{10} \simeq & -\frac{2 e I_{a c}}{C \omega^{2}}-\frac{b_{10}}{R C \omega}-\frac{2 e I_{J}}{C \omega^{2}}\left\{\cos \phi_{0} J_{1}\left(a_{-11}\right)\left[\prod_{(k, l) \neq(-1,1)} J_{0}\left(a_{k l}\right)\right]\left[\prod_{m, n} J_{0}\left(b_{m n}\right)\right]\right. \\
& \left.+\cos \left(\phi_{0}-\pi / 2\right) J_{1}\left(b_{-11}\right)\left[\prod_{(m, n) \neq(-1,1)} J_{0}\left(b_{m n}\right)\right]\left[\prod_{k, l} J_{0}\left(a_{k l}\right)\right]\right\}, \\
b_{10} \simeq & \frac{a_{10}}{R C \omega}-\frac{2 e I_{J}}{C \omega^{2}}\left\{\sin \phi_{0} J_{1}\left(a_{-11}\right)\left[\prod_{(k, l) \neq(-1,1)} J_{0}\left(a_{k l}\right)\right]\left[\prod_{m, n} J_{0}\left(b_{m n}\right)\right]\right. \\
& \left.+\sin \left(\phi_{0}-\pi / 2\right) J_{1}\left(b_{-11}\right)\left[\prod_{(m, n) \neq(-1,1)} J_{0}\left(b_{m n}\right)\right]\left[\prod_{k, l} J_{0}\left(a_{k l}\right)\right]\right\}, \\
a_{01} \simeq & -\frac{b_{10}}{R C \omega_{0}}+\frac{2 e I_{J}}{C \omega_{0}^{2}} \cos \phi_{0}\left[\prod_{(k, l)} J_{0}\left(a_{k l}\right)\right]\left[\prod_{m, n} J_{0}\left(b_{m n}\right)\right], \\
b_{01} \simeq & \frac{a_{10}}{R C \omega_{0}}+\frac{2 e I_{J}}{C \omega_{0}^{2}} \sin \phi_{0}\left[\prod_{(k, l)} J_{0}\left(a_{k l}\right)\right]\left[\prod_{m, n} J_{0}\left(b_{m n}\right)\right], \\
a_{-11} \simeq & -\frac{b_{10}}{R C\left(\omega_{0}-\omega\right)}-\frac{2 e I_{J}}{C\left(\omega_{0}-\omega\right)^{2}}\left\{\cos \phi_{0} J_{1}\left(a_{10}\right)\left[\prod_{(k, l) \neq(1,0)} J_{0}\left(a_{k l}\right)\right]\left[\prod_{m, n} J_{0}\left(b_{m n}\right)\right]\right. \\
& \left.+\cos \left(\phi_{0}-\pi / 2\right) J_{1}\left(b_{10}\right)\left[\prod_{(m, n) \neq(1,0)} J_{0}\left(b_{m n}\right)\right]\left[\prod_{k, l} J_{0}\left(a_{k l}\right)\right]\right\},
\end{aligned}
$$

and 


$$
\begin{aligned}
b_{-11} \simeq & \frac{a_{10}}{R C\left(\omega_{0}-\omega\right)}-\frac{2 e I_{J}}{C\left(\omega_{0}-\omega\right)^{2}}\left\{\sin \phi_{0} J_{1}\left(a_{10}\right)\left[\prod_{(k, l) \neq(1,0)} J_{0}\left(a_{k l}\right)\right]\left[\prod_{m, n} J_{0}\left(b_{m n}\right)\right]\right. \\
& \left.+\sin \left(\phi_{0}-\pi / 2\right) J_{1}\left(b_{10}\right)\left[\prod_{(m, n) \neq(1,0)} J_{0}\left(b_{m n}\right)\right]\left[\prod_{k, l} J_{0}\left(a_{k l}\right)\right]\right\} .
\end{aligned}
$$

Above Fourier components are in general the most important ones. They can be evaluated approximately be letting $J_{0}\left(a_{k l}\right) \simeq J_{0}\left(b_{m n}\right) \simeq 0$. Others can be computed analogously. We show in Fig. 1 how a's vary with $I_{a c}$ under the conditions $\omega=10 / R C=2 \omega_{J}=2 \sqrt{2 e I_{J} / C}$. Readers can evaluate $b_{r s}$ approximately by multiplying the corresponding $a_{r s}$ by the factor $1 / R C \omega$. The figure clearly shows that $a_{10}$ which is roughly proportional to $-2 e I_{a c} / C \omega^{2}$ is the largest in magnitude. Other components decrease rapidly with increasing subscript magnitudes.

$\omega_{0}$ can also be solved for by collecting the timeindependent terms. In fact it is easier to calculate $I_{d c}$ for a given $\omega_{0}$. One easily finds that for small $I_{J}$, $\omega_{0} \simeq 2 e R I_{d c}$ which obeys the Ohm law. In summary, the form in eq. (3) is a fast converging series and the truncation method is a good approximation.

\section{$\S 3 . \quad$ Constant-Voltage Steps}

Much interst has been concentrated on the constantvoltage steps 3 . In this section we calculate the step sizes under harmonic and subharmonic conditions. They occur when $\omega$ and $\omega_{0}$ are commensurable:

$$
q \omega=p \omega_{0}
$$

where $p$ and $q$ are non-negative integers. Now $\dot{\phi}$ is periodic in time. The time-average of $\dot{\phi}$ is

$$
\langle\dot{\phi}\rangle=2 e V_{d c}
$$

where $V_{d c}$ is the measured $d c$ voltage. Different initial conditions $\left(\phi_{0}\right)$ give different values of supercurrent and the step size is equal to the amplitude of variation. For a given $I_{d c}$, the supercurrent is equal to

$$
I_{J} \sin \phi=I_{J} \sum_{\alpha, \beta}\left[\prod_{(k, l)} J_{\alpha_{k l}}\left(a_{k l}\right)\right]\left[\prod_{m, n} J_{\beta_{m n}}\left(b_{m n}\right)\right]
$$

$$
\begin{aligned}
& \times \sin \left\{\phi_{0}+\sum_{m, n} \beta_{m n} \pi / 2\right. \\
& +\sum_{k, l, m, n}\left[\left(\alpha_{k l} k+\beta_{m n} m\right) \omega t\right. \\
& \left.\left.+\left(\alpha_{k l} l+\beta_{m n} n+1\right) \omega_{0} t\right]\right\} .
\end{aligned}
$$

Therefore, the time-average or the $d c$ part of the current requires

$$
\begin{aligned}
& \sum_{k, l, m, n}\left(\alpha_{k l} k+\beta_{m n} m\right)=-Q \\
& \sum_{k, l, m, n}\left(\alpha_{k l} l+\beta_{m n} n\right)+1=P,
\end{aligned}
$$

and

$$
\frac{P}{Q}=\frac{p}{q}
$$

where $P$ and $Q$ are the multiples of $p$ and $q$ respectively. Since larger $P$ and $Q$ implies larger indicies of Bessel functions and smaller magnitudes of super current, we discuss only the case $P=p$ and $Q=q$.

The more prominant steps are those with $p=1$. According to eqs. (20) and (21), the most important term of the supercurrent is that with

$$
\alpha_{k l}=\beta_{m n}=0
$$

for all $k, l, m$ and $n$ except for $a_{10}$ and $b_{10}$ with the requirement

$$
\alpha+\beta_{10}=-q .
$$

The time-average of the supercurrent is

$$
\begin{aligned}
\left\langle I_{J} \sin \phi\right\rangle= & (-1)^{q} I_{J} \sum_{s} \sin \left(\phi_{0}+s \pi / 2\right) J_{s}\left(b_{10}\right) J_{q-s}\left(a_{10}\right)\left[\prod_{(k, l) \neq(1,0)} J_{0}\left(a_{k l}\right)\right] \\
& \times\left[\prod_{(m, n) \neq(1,0)} J_{0}\left(b_{m n}\right)\right] .
\end{aligned}
$$

If $a_{10} \gg b_{10}$

$$
\left\langle I_{J} \sin \phi\right\rangle \simeq(-1)^{q} I_{J} \sin \phi_{0} J_{q}\left(a_{10}\right)\left[\prod_{(k, l) \neq(1,0)} J_{0}\left(a_{k l}\right)\right]\left[\prod_{(m, n)} J_{0}\left(b_{m n}\right)\right],
$$

and if $a_{10} \ll b_{10}$ 


$$
\left\langle I_{J} \sin \phi\right\rangle \simeq(-1)^{q} I_{J} \sin \left(\phi_{0}+q \pi / 2\right) J_{q}\left(b_{10}\right)\left[\prod_{(k, l)} J_{0}\left(a_{k l}\right)\right]\left[\prod_{(m, n) \neq(1,0)} J_{0}\left(b_{m n}\right)\right] .
$$

The magnitude of the right hand side is the step size. Equation (25), in many cases, is not similar to the wellknown Bessel function behavior in the voltage-source model

$$
I_{J} J_{q}\left(\frac{2 e V_{a c}}{\omega}\right)
$$

which is given by substituting

$$
\dot{\phi}=2 e\left(V_{d c}+V_{a c} \cos \omega t\right)
$$

into $I_{J} \sin \phi$. There are two reasons. First of all, $a_{10}$ does not vary linearly with $V_{a c}$ or $I_{a c}$ though the difference may be small (eq. (11)). More importantly, the factor $J_{0}\left(b_{10}\right)$ also provides considerable variation with respect to $I_{a c}$ because $b_{10}$ may not be small (eq. (12)). One can clearly see that only when eqs. (26) and (27) are applicable the familiar Bessel function behavior appears. This can explain why experimental data and simulation results are not consistent with the simple Bessel function behavior. Our calculation also shows that the oscillation amplitudes of the step sizes decrease with increasing $\omega$ or $\omega_{0}$. This is because increasing frequencies imply smaller magnitudes of $a_{r s}$ and $b_{r s}((r, s) \neq(1,0))$ and hence, smaller nonlinearity and steps.

We illustrate in Figs. 2 the functional relation of the steps sizes with the square root of the $a c$ power input. Note that there is always a proportional constant difficult to be determined. It can be viewed as an ad-

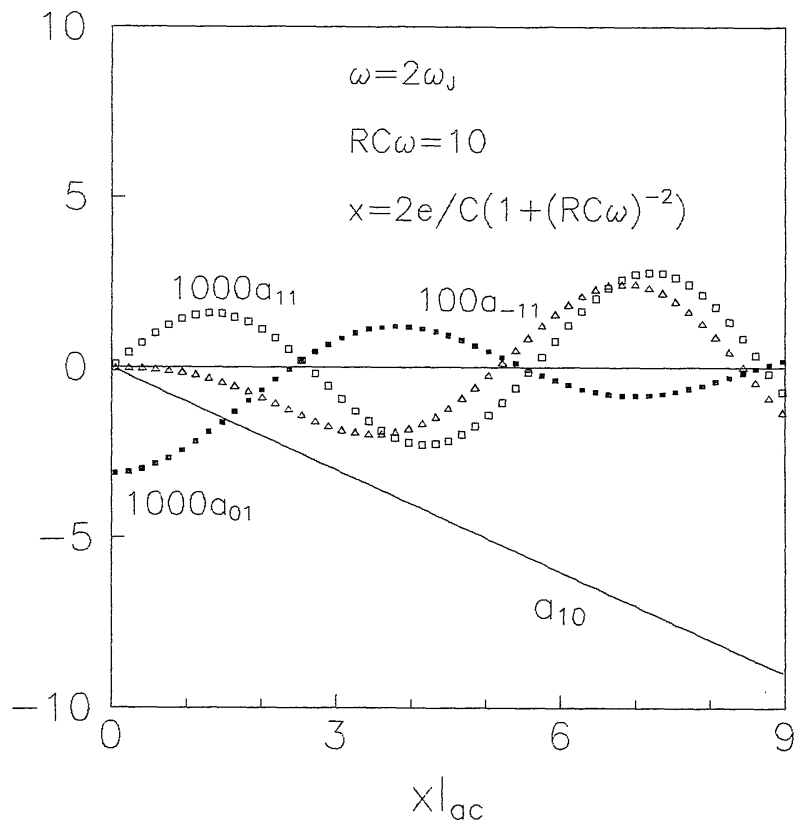

Fig. 1. $a_{10}, a_{01}, a_{-11}$ and $a_{02}$ were plotted against the squre root of input ac power: $2 e I_{a c} / C\left[1+(R C \omega)^{-2}\right]$ with $\omega=2 \omega_{J}$ and $R C \omega=10$. Note that $a_{-11}$ is multiplied by a factor of 100 and $a_{01}$ and $a_{02}$ by 1000 . justable parameter in data analysis. In our calculation the obvious choice of the variable for the case $R C \omega \gg 1$ is $2 e I_{a c} / C\left[1+(R C \omega)^{-2}\right] \simeq a_{10}$. Because in this case $\left|a_{10}\right| \gg\left|b_{10}\right|$ and hence, the variation of the averaged current comes mostly from that of $a_{10}$. If, however, $R C \omega \ll$ $1,\left|a_{10}\right| \ll\left|b_{10}\right|$, then the variation of $b_{10}$ has more important impact and $2 e I_{a c} / R C^{2} \omega\left[1+(R C \omega)^{-2}\right] \simeq b_{10}$ should be chosen. In Fig. 2 we choose $\omega=2 \omega_{J}$ and $R C \omega=10$ in Fig. 2(a) and $R C \omega=1$ in Fig. 2(b). Three step sizes were shown, namely $q=0$ in the solid lines, $q=1$ in the
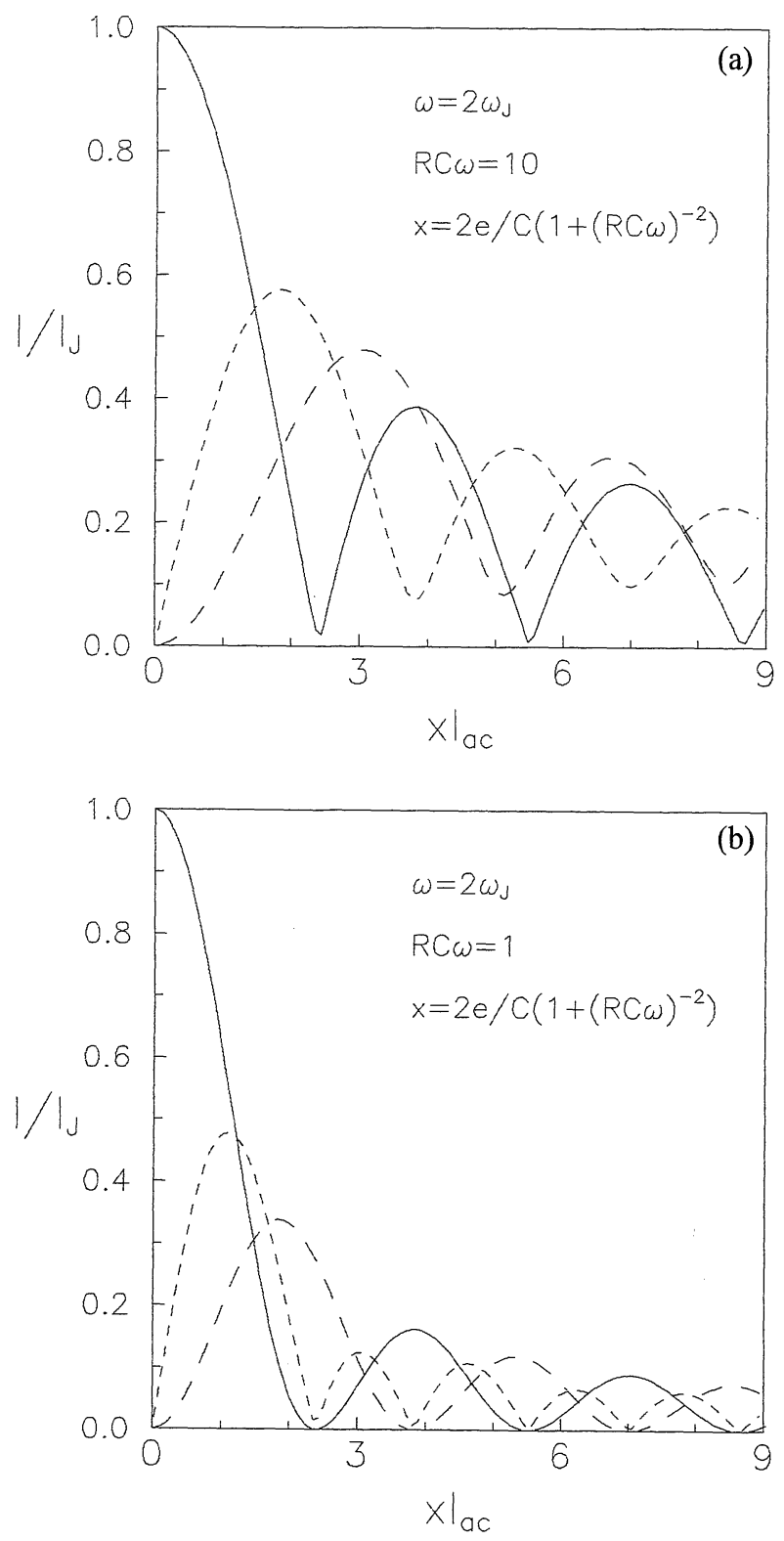

Fig. 2. The measured $d c$ supercurrent versus $2 e I_{a c} / C[1+$ $\left.(R C \omega)^{-2}\right]$ : the solid line denotes $\omega_{0}=2 e V_{d c}=0$, the short dashed line $\omega=\omega_{0}$ and the long dashed line $2 \omega=\omega_{0}$ under the condition (a) $\omega=2 \omega_{J}$ and $R C \omega=10$ and (b) $\omega=2 \omega_{J}$ and $R C \omega=1$. 
short-dashed lines and $q=2$ in the long-dashed lines. One would observe that the curves in Fig. 2(a) are close to Bessel functions. In fact they can be approximated by

$$
J_{q}\left(a_{10}\right) \simeq J_{q}\left(\frac{2 e I_{a c}}{C\left[1+(R C \omega)^{-2}\right]}\right),
$$

as we have seen in eq. (26). Those in Fig. 2(b) are quite different from the Bessel functions. Now $a_{10}=b_{10}$, so the variation of $b_{10}$ is significant. These curves are approximately

$$
J_{q}^{2}\left(\frac{2 e I_{a c}}{C\left[1+(R C \omega)^{-2}\right]}\right)
$$

for $q=1$ and

$$
J_{0}\left(\frac{2 e I_{a c}}{C\left[1+(R C \omega)^{-2}\right]}\right) J_{q}\left(\frac{2 e I_{a c}}{C\left[1+(R C \omega)^{-2}\right]}\right)
$$

for $q=2$. Analogously, for $R C \omega \ll 1$, the step size should be close to

$$
J_{q}\left(b_{10}\right) \simeq J_{q}\left(\frac{2 e I_{a c}}{R C^{2} \omega\left[1+(R C \omega)^{-2}\right]}\right)
$$

in view of eq. (27).

For $p \neq 1$, the subharmonic steps, the step size is considerably smaller. The most important terms of the supercurrent are

$$
\begin{aligned}
\left\langle I_{J} \sin \phi\right\rangle= & (-1)^{q} I_{J} \sum_{m, n} \sin \left[\phi_{0}+\sum(n-m) \pi / 2\right] \\
& \times J_{q-m}\left(a_{10}\right) J_{m}\left(b_{10}\right) J_{p-n-1}\left(a_{01}\right) J_{n}\left(b_{01}\right) \\
& \times\left[\prod_{k, l}^{\prime} J_{0}\left(a_{k l}\right)\right]\left[\prod_{m, n}^{\prime} J_{0}\left(b_{m n}\right)\right] \\
& +J_{q+p-1-m}\left(a_{10}\right) J_{m}\left(b_{10}\right) \\
& \times J_{p-n-1}\left(a_{-11}\right) J_{n}\left(b_{-11}\right) \\
& \times\left[\prod_{k, l}^{\prime \prime} J_{0}\left(a_{k l}\right)\right]\left[\prod_{m, n}^{\prime \prime} J_{0}\left(b_{m n}\right)\right]
\end{aligned}
$$

where $\Pi^{\prime}$ and $\Pi^{\prime \prime}$ mean that $(k, l)$ and $(m, n)$ are not equal to $(1,0),(0,1)$ and $(1,0),(-1,1)$ respectively. Since $a_{-11}, b_{-11}$ and $a_{01}, b_{01}$ are comparable in magnitude, there are two kinds of terms contributing to the $d c$ supercurrent. Hence, the relation between the step sizes and $I_{a c}$ are very complicated. In Fig. 3 under the conditions $\omega=2 \omega_{J}$ and $R C \omega=10$, we show the steps labeled by $(p, q)$. There are still oscillations but the minima do not approach zero. Their positions are shifted slightly away from the zeroes of Bessel functions. Furthermore, our calculation shows that the larger the magnitude of $p$ or $q$, the smaller the step size. This is expected. But our calculation also shows that when $|p-q|=1$, the magnitudes of $a_{-11}$ and $b_{-11}$ are the largest, and hence, the step sizes are greater.

\section{§4. Conclusion}

We have proposed an analytical solution for the RSJ model. It shows Ohmic behavior in general. When $\omega$ and $\omega_{0}=2 e V_{d c}$ are commensurable the solution becomes a

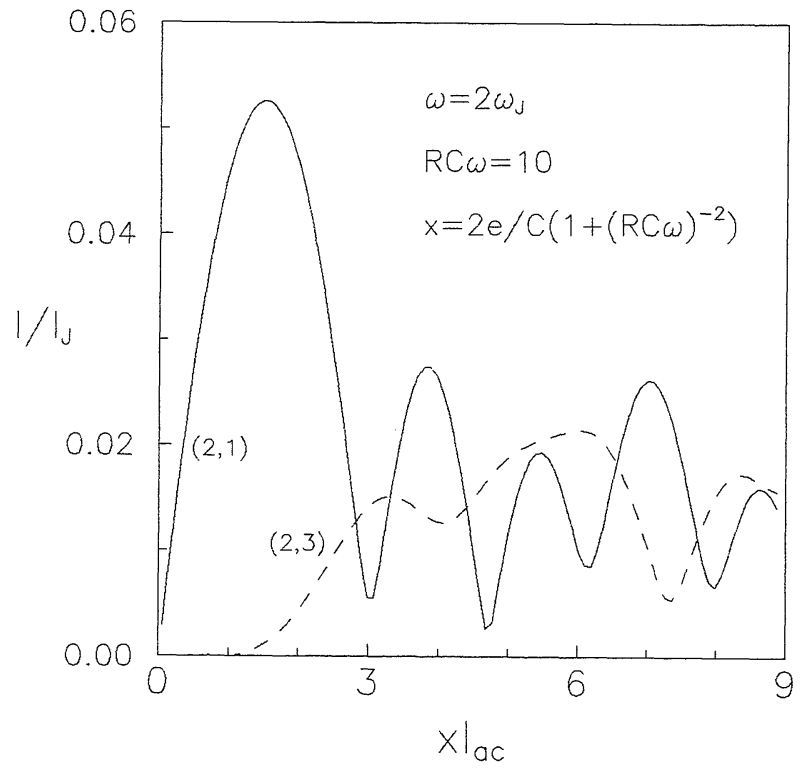

Fig. 3. The measured $d c$ supercurrent versus $2 e I_{a c} / C[1+$ $\left.(R C \omega)^{-2}\right]$ : the solid line denotes $\omega=2 \omega_{0}$ and the dashed line $3 \omega=2 \omega_{0}$ under the condition $\omega=2 \omega_{J}$ and $R C \omega=1$.

periodic function and produces constant-voltage steps. The step size is a function of the square root of the input ac power. For the harmonic case, i.e. $n \omega=\omega_{0}$, only if $R C \omega \ll 1$ and $R C \omega \gg 1$, the step size has the well-known Bessel function form. Otherwise, the magnitude of step size cannot be described by Bessel functions though there are clearly oscillations. For the subharmonic case, i.e. $p \omega=q \omega_{0}$, the step size still shows oscillations but without definite amplitude. The minima occur approximately at the zeroes of Bessel functions.

This work is supported in part by the NSC of Taiwan, R.O.C. under the contract number NSC86-2112-M-002005 .

\section{Appendix}

In this appendix we give some details of deriving eqs. (6) and (7). In view of eqs. (3)-(5), we found

$$
\begin{aligned}
\mathrm{e}^{i \phi}= & \mathrm{e}^{i\left(\phi_{0}+\omega_{0} t\right)}\left[\prod_{k, l} \sum_{p} J_{p}\left(a_{k l}\right) \mathrm{e}^{i p\left(k \omega+l \omega_{0}\right) t}\right] \\
& \times\left[\prod_{m, n} \sum_{q} J_{q}\left(b_{m n}\right) \mathrm{e}^{i q\left[\left(m \omega+n \omega_{0}\right) t+\pi / 2\right]}\right]
\end{aligned}
$$

where $p$ and $q$ are integers. After some arrangement, we got

$$
\begin{aligned}
\mathrm{e}^{i \phi}= & \mathrm{e}^{i\left(\phi_{0}+\omega_{0} t\right)} \sum_{\alpha_{k l}}\left(\prod_{k, l} J_{\alpha_{k l}}\left(a_{k l}\right) \exp \left[i \alpha_{k l}\left(k \omega+l \omega_{0}\right) t\right]\right) \\
& \times \sum_{\beta_{m n}}\left(\prod_{m, n} J_{\beta_{m n}}\left(b_{m n}\right)\right. \\
& \left.\quad \times \exp \left\{i \beta_{m n}\left[\left(m \omega+n \omega_{0}\right) t+\pi / 2\right]\right\}\right)
\end{aligned}
$$

where $\alpha$ and $\beta$ are also integers. Substituting eqs. (3) and (A.2) into eq. (2) and collecting the coefficients of 
$\sin \left(r \omega t+s \omega_{0} t\right)$ and $\cos \left(r \omega t+s \omega_{0} t\right)$ seperately, we obtain eqs. (6) and (7) with constraints eqs. (9) and (10).

Equation (8) was obtained by requiring $r=s=0$.

1) Recent works can be found in Macroscopic Quantum Phenomena and Coherence in Superconducting Networks (World Scientific, Singapore, 1995); Proc. 2nd European Conf. on Applied Superconductivity (EUCAS), 1995 (IOP. Bristol UK, 1995). Other recent related works are A. Kenfack and T. C. Kofane: Phys. Rev. B 52 (1995) 10359; C. B. Whan and C. J. Lobb: Phys. Rev. B 53 (1996) 405 and L.-M. Kuang, Y. Wang and M.-L. Ge: Phys. Rev. B 53 (1996) 11764.

2) S. Shapiro: Phys. Rev. Lett. 11 (1963) 80.

3) For a review, see A. Barone and G. Paterno: Physics and
Applications of the Josephson Effect (John Wiley and Sons, New York, 1982).

4) D. E. McCumber: J. Appl. Phys. 39 (1968) 2503; ibid. 3113; W. C. Stewart: Appl. Phys. Lett. 12 (1968) 277.

5) V. N. Belykh, N. F. Pedersen and O. H. Soerensen: Phys. Rev. B 16 (1977) 4853; ibid. 4860.

6) One of the early important analytical works is A. Lee Patrick: J. Appl. Phys. 42 (1971) 325.

7) V. A. Pliss: Nonlocal Problems of the Theory of Oscillations (Academic, New York, 1966).

8) L.-E. Hasselberg, M. T. Levinsen and M. R. Samuelsen: Phys. Rev. B 9 (1974) 3757; J. Low Temp. Phys. 21 (1975) 567.

9) C. D. Hu: Phys. Rev. B 54 (1996) 10065.

10) P. M. Morse and H. Feshbach: Methods of Theoretical Physics (McGraw-Hill, New York, 1978) Chap. 5. 